\title{
Kāyānupassanā: si de yoga budista se trata
}

Eduardo Francisco Freyre Roach

\section{Resumen}

En este artículo se exploran sutras budistas tempranos (del Canon Pali) donde se expone la práctica del mindfulness, o de la contemplación plena: Satipaţ̧hāna Sutta, Mahā Satipaţ̧hāna Sutta, y Ānāpānasati Sutta. Se coloca el énfasis en la práctica yoga Kāyānupassanā (mindfulness del cuerpo), sus variantes y objetivos, fundamentos, y la visión de la corporeidad en que se sustenta. También se le compare con las doctrinas y técnicas yogas que se exponen en el Bhagavad Gita y los Yoga Sutras de Patanjali. Y se muestra como a pesar de su destinación soteriológica, el mindfulness yoga budista esta siendo "aplicado", no sin algunos riesgos, en el mejoramiento del rendimiento deportivo de los atletas.

Palabras clave: Yoga Budista, Mindfulness, Cuerpo, Educación Física, Deportes.

\footnotetext{
* Licenciado e Doutor em Ciências Filosóficas pela Universidad Estatal de Moscú - Rússia. Mestrado em estudos Budistas pela Universidade de Hong Kong - China. E-mail: freyre.roach2007@gmail.com
} 


\section{Kāyānupassanā: si da ioga budista se- -tratase}

Resumo

Este artigo explora três sutras budistas em sutras budistas precoce (do Cânone Pali), onde é exposta a prática do mindfulness ou contemplação completa. Satipaţ̧̧hāna Sutta, Satipaţ̧̦hāna Sutta Mahā, and Ānāpānasati Sutta. Coloca-se a ênfase na prática ioga Kāyānupassanā ("mindfulness do corpo"), suas variantes e objetivos, fundamentos e a visão da corporeidade em que se baseia. Também se - compara com as doutrinas e técnicas iogas Bhagavad Gita e de loga Sutras de Patanjali. Mostra-se como apesar de seu destino soterológica, o mindfulness budista está sendo "aplicada", não sem alguns riscos, no campo do melhoramento do rendimento esportivos do atleta.

Palavras Chave: loga Budista, Mindfulness, Corpo, Educação Física, Esportes.

\section{Kāyānupassanā: if it is about the buddhist yoga}

\section{Abstract}

This article explores three early Buddhist sutras (from the Pali Canon), where it is exposed the practice of the mindfulness, or full contemplation: Satipațţhāna Sutta, Mahā Satipațţhāna Sutta, and Ānāpānasati Sutta. It emphasises on practice yoga Kāyānupassanā (mindfulness of the body), its variants and objectives, foundations, and the vision of the corporeality on which it is based. It is compared with the yoga doctrines and techniques exposed in Bhagavad Gita and Yoga sutras of Patanjali. It shows that despite its soteriological destination, the Buddhist mindfulness is being "applied", not without some risks, in the field of physical education and sports training.

Key Words: Buddhist yoga, Mindfulness, Corporeality, Physical Education, Sports. 


\section{Introducción}

El término de "yoga" es de factura hindú. Inicialmente yoga significa unión o fusión alma-cuerpo y alma humana-Dios Brahma. Un "yogui" seria aquel que practica este camino "espiritual" o "religión". Tal sentido general de la palabra yoga se le puede trazar en las escrituras antiguas sagradas de la India, sobre todo en Upanishad y Mahabharata. Yoga también alude a prácticas donde se combinan posturas y movimientos corporales, ejercicios respiratorios y meditación, en función del cultivo de ciertas cualidades corporales y estados mentales. En el Bhagavadgita (Bhagavad Gita o simplemente Gita), uno de los libros del Mahabharata, este sentido técnico subsidia al sentido general de yoga.

Los sutras tempranos del Budismo (Canon Pali) también informan de técnicas yogas. Es el caso de Kāyānupassanā: la contemplación o meditación del cuerpo en el cuerpo simplemente atención plena del cuerpo o "mindfulness" del cuerpo. Este artículo explora en qué consiste este yoga, sus variantes, objetivos, visión de la corporeidad humana en que se sustenta, su ruptura con las doctrinas yogas del Gita y Patanjali, y, por ultimo, su aplicación en el campo de Mindful Sport Performance Enhancement $(\mathrm{MSPE})^{1}$

\section{Kāyānupassanā, sus variantes}

Hay dos sutras que se consideran clásicos textos yogas o de meditación en el Budismo: Satipaţ̧̧hāna Sutta (MN. 10) y Mahā Satipaţţhāna Sutta (DN. 22) ${ }^{2}$. Sati, del Pali se ha traducido preferentemente al inglés como "Mindfulness" $^{\prime \prime 3}$ (THE SATIPATTHANA SUTTA, 2003) y al español como "Atención Plena" (NHAT, 1999) o simplemente "Atención" (MAJJHIMA NIKAYA, 1999, p.113). A groso modo mindfulness seria estar completamente atento o consciente a lo que sucede en el momento presente, suspendiendo todo juicio e intervención.

En estos sutras se describen cuatro prácticas: Kāyānupassanā, Vedanānupassanā, Cittānupassanā, y Dhammānupassanā, respectivamente, mindfulness del cuerpo, de las sensaciones, de la mente (consciencia), y de la doctrina de Buda de las Cuatro Nobles Verdades
(Cattāri Ariya Saccāni). A la enseñanza contenida en los sutras budistas MN.10 y DN. 22 del Canon Pali budista suele llamársele Cattadro Satipaţ̧hāna (Fundamentos de la Atención Plena). Cuatro son esos fundamentos u objetos del mindfulness: kāyā (cuerpo), vedanā (sensación o emoción), cittā (mente), y dhammā (doctrina de Buda). Kāyānupassanā se refiere al cuerpo. El sutra MN. 119 (KAYAGATA-SATI SUTTA, 2013) despliega con mas amplitud esta práctica. Estos sutras presentan cuatro variantes de este mindfulness.

En primer lugar se tiene el mindfulness sobre la respiración. Lo primero que se orienta es estar sentado en un lugar tranquilo y con las piernas cruzadas, de manera que la columna vertebral se encuentre erecta y flexible. Así se consigue que el cuerpo se mantenga por largo tiempo erguido, equilibrado, y flexible. Se alude aquí pādāsana (Lotus), postura corporal ancestralmente asumida por los meditadores, y que se ha integrado a la cultura común y corriente de sentarse en el hemisferio oriental. Tal práctica de ānāpānasati se presenta en el MN. 10 de la siguiente forma:

De la misma manera que un tornero experto o su aprendiz sabe, al labrar una voluta grande: "Estoy labrando una voluta grande", o al labrar una voluta corta, sabe: "Estoy labrando una voluta corta", así el monje, al hacer una aspiración larga, sabe: "Hago una aspiración larga". Al hacer una aspiración corta, sabe: "Hago una aspiración corta". "Consciente de todo el cuerpo (respiración), aspiraré", así se ejercita. "Consciente de todo el cuerpo (respiración), espiraré", así se ejercita. Calmando la actividad corporal (respiración)' aspiraré, así se ejercita. Calmando la actividad corporal (respiración), espiraré, así se ejercita. Así vive contemplando el cuerpo en el cuerpo interna y externamente. Vive contemplando los factores del origen del cuerpo, o vive contemplando tanto los factores de origen como los de disolución del cuerpo. O tiene consciencia de que "hay un cuerpo" en el grado necesario para el conocimiento y la atención, y vive con independencia, sin apegarse a nada en el mundo. Monjes, así es como un monje vive contemplando el cuerpo en el cuerpo (MAJJHIMA NIKAYA, 1999, P. 117).

Sucintamente seria prestar atención la respiración tal como está aconteciendo. Muy importante es destacar que todo jui- 
cio se suspende. Hay que dejar que la respiración fluya. No se contempla interrumpir ni la aspiración ni la exhalación.

Debe aclararse que el texto no habla ni del orden ni el tiempo de duración de estos ejercicios meditativos. Todo depende del criterio que adopte los practicantes y los guías de meditación, sea en los templos o monasterios, o los centros de meditaciones, u otros contextos. Sin embargo, es de suponer que la atención a la respiración resulta un buen comienzo para quienes se inician en la práctica del mindfulness. Al mismo tiempo se trata de un objeto de meditación de fácil acceso a cualquier grado de cultura meditativa. Usted puede estar parado, acostado, o caminando como en el caso de la usual practica de "warking meditation" (meditación caminando) ${ }^{4}$ en la tradición Zen del Master budista vietnamita Thich Nath Hahn. También se recomienda algo que indique interrumpir por unos segundos lo que se está haciendo para meditar en la respiración. Es el caso de un programa (software) como la "mindfulness Bell"5 (La Campana del Mindfulness) instalado a su computadora, que le recuerde volver a la atención a la respiración, aunque sea por menos de un minuto. Algunos master budistas aconsejan visualizarse como respira un niño durmiendo, lo se conoce como "baby breathing meditation".

En el MN.10 y el DN. 22) se habla, en segundo lugar, de la variante de la atención a las posturas del cuerpo. Es decir, uno sabe y se dice a uno mismo "estoy caminando", "estoy de pie", "estoy sentado", "estoy acostado". Y así mismo en cualquier otra posición corporal o actividades corporales tales como "avanzar", "retroceder", "mirar hacia delante", "mirar alrededor", "extender los miembros", "encoger los miembros", "vestirse", "comer", “"beber", etc.

Se prescribe la meditación en cada parte del cuerpo, órganos, y tejidos, así como las llamadas impurezas del cuerpo: fluidos corporales como las heces, bilis, flemas, pus, sangre, sudor, grasa sólida, lágrimas, grasas líquidas, saliva, mocos, fluido sinovial, y orina. Esta tercera variante contempla también el mindfulness de los elementos materiales que componen el cuerpo: tierra, agua, aire y fuego.

Finalmente el discurso alude a una cuarta variante que consiste en la atención plena de un cadáver en su proce- so de descomposición y putrefacción. Entonces uno ha de decirse: "Es verdad que este cuerpo mío tiene también la misma naturaleza, se volverá igual y no escapará a ello" (MAJJHIMA NIKAYA, 1999, p. 134).

Kāyānupassanā da la impresión como si el cuerpo se entendiera solo en su dimensión anatómica, fisiológica, y temporal, y como algo que no es atractivo, más bien repulsivo o repugnante, del cual hay que desapegarse, ya sea con su mortificación o el suicidio. Sin embargo, hay que tener que este mindfulness se hace también respecto a aspectos como las sensaciones y los objetos mentales, es decir, el cuerpo en su dimensión sensorial o psíquica. De hecho las partes de los sutras que tratan del mindfulness de las sensaciones y los objetos mentales se refieren a esta dimensión de la corporeidad humana. Uno puede observar que en el fondo se muestra una línea de gradación en la práctica, desde los aspectos físicos más visibles a los aspectos sensoriales no visibles.

Además, en el Ānāpānasati Sutta (MN. 118) se indica:

\begin{abstract}
Yo os digo - se dice en este sutra- que una de las contemplaciones del cuerpo en el cuerpo es la contemplación a la respiración...Yo os digo que una de las contemplaciones de las sensaciones en las sensaciones es la contemplación en la respiración...Así es, monjes como la atención a la respiración, cultivada y practicada con asiduidad, lleva a la perfección de los cuatro fundamentos de la atención (MAJJHIMA NIKAYA, 2006, p.132-134).
\end{abstract}

Es decir, que el mindfulness de la respiración se aplica a todos los objetos ${ }^{6}$. Esta presente cuando aparece la sensación de éxtasis o rapto, pasión y apego en la meditación.

Segundo, no se pierda de vista cuando se habla de impurezas del cuerpo, no es que se este juzgando a esos supuestos objetos (los fluidos). Simplemente, que el meditador este atento ("mindfull") tanto al objeto que se considera impuro, como a la misma sensación de repugnancia que el objeto le pueda sentir. Cabe reiterar que resulta esencial al mindfulness que el meditador mantenga a raya sus apreciaciones subjetivas, predisposición, proclividades, y reacción hacia la experiencia que 
esta teniendo. Es decir, no se trata de decir si el cuerpo es no es intrínsicamente bello o feo. Mas bien el asunto es que el meditador modificar la experiencia de tener cuerpo y su visión de la naturaleza mismo, en el sentido que se explicara mejor mas adelante. Por ultimo, merece puntualizarse que el desapego al cuerpo no se toma en stricto sensu. Al fin y al cabo lo que se espera lograr con el mindfulness es que el meditador abandone las asunciones tanto negativas como positivas sobre el cuerpo ${ }^{7}$.

\section{la meta soteriologica de Kāyānupassanā}

En el sutra MN.119 se listan diez beneficios del mindfulness del cuerpo:

Uno supera lo que desagrada y agrada....Uno soporta el frío y el calor, el hambre y la sed,... las formas de hablar hostiles e importunas,... el surgir de las sensaciones corporales dolorosas...Uno oye tanto los sonidos humanos como los divinos... Uno obtiene diversas facultades paranormales... Uno percibe con su mente la mente de otros seres...Uno recuerda sus múltiples vidas anteriores...Uno, habiendo experimentado por su propio conocimiento superior en este mismo mundo la aniquilación de las corrupciones, alcanza y permanece en la liberación de la mente y la liberación por la sabiduría que están libres de corrupciones (MAJJHIMA NIKAYA, 2006, p.142-144)

Leyendo el sutra MN. 10 es posible darse cuenta que el último beneficio es la meta fundamental, lo cual se expresa en la siguiente sentencia que se repite en los sutras budistas sobre mindfulness:

Monjes, el único camino para la purificación de los seres, para la superación de la pena y las lamentaciones, para la destrucción del dolor y la aflicción, para alcanzar el recto sendero, para la realización del Nibbana, es éste: Los Cuatro Fundamentos de la Atención (MAJJHIMA NIKAYA, 2006, p.116).

En efecto, tal declaración marca la finalidad del mindfulness budista. No debe perderse de vista que el Budismo ante todo contempla una religión, y es de las que postulan una creencia y teoría de la salvación humana (Soteriología). Sin embargo, la religión del Budismo no es teís- ta, es decir, no se compromete con la creencia en Dios o deidades transcendentes ni mucho menos que hay alma sin cuerpo.

Ahora bien, la Soteriología del Budismo se sustenta en la doctrina Cattäri ariyasaccāni (Cuatro Nobles Verdades). Aunque no es de las más tempranas, ha devenido en la doctrina que identifica ancestralmente al Budismo. Al menos, si bien hay contrapunteos entre vertientes del Budismo, respecto a esta doctrina existe gran consenso. De hecho en los sutras budistas reiteradamente se presenta como la Buddhavacana (palabra de Buda) y la frontera que marca la distancia entre el Budismo y otras tradiciones espirituales.

A groso modo, la Primera Noble Verdad (Dukkham Ariyasaccam) postula la existencia de dukkha, el sufrimiento o lo insatisfactorio ${ }^{8}$. El sutra SN.56 define dukkha como lo estresante, y se lista como tal el nacimiento, el envejecimiento, la lamentación, la preocupación, el dolor, la desesperación, así como encontrarse con lo que no se desea, separarse de lo que se desea, no ser lo que se desea, etc. (DHAMMACAKKAPPAVATTANA SUTTA, 2013, p.2) ${ }^{9}$. La primera parte de este inventario de los sufrimientos tiene que ver con las experiencias que motivaron a Gautama, Gotama, o al Buda como personaje histórico a la búsqueda de las causas y la erradicación del sufrimiento. Entonces se explica porque MN.10, DN. 22, y MN. 119 presentan a kāya (el cuerpo) como el primer fundamento y a Kāyānupassanā como la primera práctica del mindfulness. Obviamente existe sintonía entre este inventario de dukkha y el mindfulness sobre la composición física y anatómica del cuerpo y su temporalidad. No es difícil darse cuenta que la práctica del mindfulness del cuerpo desarrolla la interiorización de que hay un cuerpo y de su aceptación tal y como es. La Segunda Noble Verdad (Dukkhasamudayam Ariyasaccam) reza sobre el origen o causa de dukkha. Se alude a tanhā, la sed, el deseo, la codicia, el apego o aferramiento. Los sutras se refieren a tres tipos de tanhā: Kama-tanha (sensoriales), Bhava-tanha (ser como se desea), Vibhava-tanha (no existir para evadir todo dolor). ¿Puede erradicarse el sufrimiento? La Tercera Noble Verdad (Dukkhanirodham Ariyasaccam) brinda respuesta afirmativa a esta pregunta. Aquí se habla de la posibilidad del alcanzar el estado de no sufrimiento 
mediante la supresión del desapego. Por ultimo, la Cuarta Noble Verdad (Dukkhanirodhagämanī Patipadā Ariyasaccam) concierne sobre el sendero para lograr el nibbana. Aquí se habla de Ocho Nobles Vías (Ariyo Atthangiko Maggo), factores, o prácticas correctas (sammā), adecuadas, idóneas, convenientes o ideales: "correcta visión" (Sammādivhi), "correcta sustentación" (Sammā-Ājīvo) , "correcta intención" (Sammāsankappo) ,"correcto hablar" (Sammāvācā) ,"correcto esfuerzo" (Sammāvāyāmo), "correcta concentración" (Sammāsamādhi), "correcto mindfulness" (Sammāsati) . Lo referente al correcto hablar, comportarse corporalmente, y buscar el sustento de la vida, se subsume bajo el término de silla (ética o moral). Sati (mindfulness) alude a la rectitud del esfuerzo, la concentración y el mindfulness. Paññā (sabiduría) engloba la intención y visión correctas ${ }^{10}$.

Se deduce que Kāyānupassanā es parte de sati, y del sendero que lleva al nirvana. De ahí que en la exposición del mindfulness respecto al cuerpo, varios pasajes hablan de que esa práctica conduce a la concientización de que meramente "this is body!" (jhay cuerpo!). Y en tal medida se logra la vivencia de la impermanencia e impersonalidad del cuerpo, y con ello la erradicación del desapego a todo. Precisamente una de las definiciones de nirvana, tal y como se insiste en el sutra MN. 26 (Aryaparisana sutta) es "la suprema salvación de toda sujeción" (MAJJHIMA NIKAYA, 2006, p.8).

\section{Käyānupassanā: vision de la corporiedad}

Ciertamente, Kāyānupassanā se refiere explícitamente a la meditación sobre la composición anatómica (órganos, huesos, tejidos, etc.) del cuerpo, sus elementos materiales (tierra, agua, fuego, aire), determinados procesos y fluidos fisiológicos, así como el envejecimiento corporal, y la muerte. Sin embargo, el Budismo no se compromete una visión reduccionista y dualista de la corporeidad humana. ! Veamos!

En el sutra MN. 26 se lee que el apego ocurre porque "nos bloqueamos", "nos atamos" a objetos (formas materiales, los objetos tangibles, visuales, sonidos, olores, o sabores, es lo que se denominan "las cinco sogas de los sentidos". Es decir, esos objetos nos seducen, cae- mos en su trampa, red o enredo, nos enviciamos y nos apasionamos con ellos. De forma mas técnica en los sutras budistas se suelen encontrar pasajes donde una vez que se habla de las formas de sufrimiento (primera noble verdad) se plantea como resumen y colofón que Sankhittena pañcupādānakkhandhā dukkhā, es decir, los cinco agregados del apego (khandhas) son dukkha" (DHAMMACAKKAPPAVATTANA SUTTA, 2013, p.2). Por tal se entienden: nama-rūpa (materialidad-mentalidad), vedanā (sensaciones, emociones o sentimientos), sañña (percepciones), sankhära (formaciones, construcciones, fabricaciones, proliferaciones mentales, corporales y conceptuales), y viññāna (consciencia ${ }^{11}$. Se reitera que son impermanentes y funcionan interactivamente ${ }^{12}$. "Monjes, - se plantea en el sutra MN.38 - la conciencia se define por la condición especifica de la que surge en dependencia: si la conciencia surge en dependencia del ojo y las formas visibles, se define como conciencia visual... ..."(MAJJHIMA NIKAYA, 2006, p.231). Sucesivamente se dice lo mismo respecto cuando la consciencia esta determinada por el oído y los sonidos, la nariz y los olores, la lengua y los sabores, el cuerpo y los objetos tangibles, y la mente y los objetos mentales. Co lo cual se descartan el dualismo psicofísico y el reduccionismo fisicalista de la corporeidad humana. El meditador entrenado es capaz de ver el aparecer y desvanecer de los agregados, y sus interacciones.

Obviamente, no es lo mismo cuando se toma el cuerpo como cuerpo que cuando se toma como uno de los cinco agregados del apego. Y lo mismo se aplica para las sensaciones, la consciencia y los objetos mentales que se mencionan. No es difícil notar que el grado de mindfulness de los agregados es directamente proporcional al desarrollo alcanzado en tal práctica del mindfulness del cuerpo, de las sensaciones, la conciencia, y los objetos mentales. Se explica entonces porque los sutras mencionados ofrecen un orden de exposición que va desde la meditación sobre el cuerpo hasta la meditación sobre los cinco agregados y las cuatro verdades hasta orden de explosión, pasando por la meditación sobre las sensaciones, los objetos mentales y la consciencia. Quien llega a estos niveles superiores de meditación logra tipo de meditación ha logrado un alto grado de desapego y, entre otras cosas, supera la visión de la escisión cuerpo. 
Ahora bien, sutras budistas tempranos aluden al Surgimiento Dependiente (Pratitya-samutpäda). Tal doctrina reza "Cuando esto es, eso existe, al surgir esto, eso surge..." (MAJJHIMA NIKAYA, 2006, p.234), en esencia, que no hay nada en el mundo separado o aislado, y causa de sí mismo. Todo lo que aparece y se desvanece ocurre por determinadas causas y condiciones ${ }^{13}$. Esta doctrina contempla que hay 12 factores interconectados, al surgir uno, surge el otro. Estos son: "ignorancia", "composiciones mentales", "conciencia", "organismo psicofísico", "seis esfera de los sentidos", "contacto", "deseo", "apego", "devenir", "nacer-envejecer-morir-pena-lamento-dolor-aflicción-tribulación". Y se sentencia: "He aquí el origen de todo este montón de sufrimiento" (MAJJHIMA NIKAYA, 2006, p. 234; MAHA-NIDANA SUTTA, 2013). En el mismo contexto se habla de lo podríamos decir el la "cesación dependiente", es decir, la reversión del sufrimiento. Aquí también se explica no solo que entre los agregados hay intercondicionamientos, sino que se relacionan de la misma manera con otros factores como la ignorancia, las formaciones mentales, y los factores kármicos. Y se infiere que la corporeidad humana no es más que la interconexión de los cinco agregados del apego en correspondencia con el surgimiento dependiente.

Como colofón de Pratittya-samutpāda se tiene un postulado fundamental de la filosofía del Budismo: anattā, es decir, la doctrina de que por cuanto nada es condición de sí mismo, a nada se le puede atribuir ser en sí mismo. En sutras como el Milindapañha sutta (1890), Potthapada sutta (2013) y Chachakka Sutta (2013) se explica exhaustivamente como el surgimiento dependiente asumido lleva al abandono de la asunción del ser, y, por consiguiente de la practica de autoidentificación. Esto seria interiorizar y externalizar mental, discursiva y corporalmente etam mama (esto es mío), eso ham asmi (este soy yo), eso me attā (este es mi ser en si) (MAJJHIMA NIKAYA, 2006, p.220). Por lo tanto, si se afirma que los agregados están carentes de ser, entonces eso mismo se aplica al cuerpo, a la mente, a los dolores, en fin a todos. Es decir, se erradicar la practica de "este es mi cuerpo", "soy mi cuerpo", "mi cuerpo es mi ser".

La doctrina de anattā lleva a otro postulado fundamental: śūnyatā, es decir, la vaciedad o vacuidad de ser de todo cuanto existe. El sutra MN. 121 se habla en términos de "la vacuidad de lo que allí no existe" (MAJJHIMA NIKAYA, 2006, p. 162), y como toda percepción (sea de un bosque, un pueblo, la gente, una piel de toro, la tierra, el espacio, la conciencia ilimitada, la esfera de la nada, etc.) es vacía de ser en si. Los sutras de la tradición budista Mahayana ponen el acento en esta implicación de la doctrina del surgimiento dependiente. Se desprende que el mindfulness del cuerpo tributa a esta meta, en tanto se trata que el meditador vivencie que solo hay mero cuerpo, cuando llega a la meditación de los agregados vivencia y el surgimiento dependiente, alcanza la experiencia directa del no-ser o la vacuidad del cuerpo. En virtud de esto el apego esta privado de fundamento ontológico.

El apego esencial que puede decirse esta en la base de todos los apegos es el apego al yo. Dada esta corrección entre el apego y el desapego al cuerpo y al yo, se cierra la perspectiva de asunción de los dolores del cuerpo como algo privado, y fatal o trágico.

En fin, las doctrinas budistas de los cinco agregados del apego, el surgimiento dependiente, el no ser en si mismo, y la vacuidad, sugiere una visión "monista" de la corporeidad humana. El mindfulness del cuerpo traduce este enfoque a una dimensión práctica.

\section{Kāyānupassanā en una perspectiva comparativa}

Recientemente se ha establecido que en el Gita el yoga en sentido técnico aparece preferentemente subsumido en otros términos como diksa, dhyana, tapas, brahmacarya y upasana (ENCYCLOPEDY OF HINDUISM, 2011, p. 596). En los marcos de esta misma tradición vedica se encuentran los Yoga Sutras (Yogasūtra) de Patañjali o Patanjali. Considerada como clásica literatura del yoga, en esta obra se ofrece un registro sin precedente de técnicas yogas.

Los sutras del Canon Pali del Budismo, cronológicamente anteriores (NATH, 2008), informan de técnicas yogas, pero no con tanta recurrencia y explicites como se puede verificar en el Gita y los yogas sutras de Patanjali. Estos testimonios escritos, aunque comparten temas y términos, no se mencionan entre si. ¿Aquellos influyeron en estos o viceversa? El debate sobre esta cuestión sigue en 
pie (FRAWLEY, 2012). Sin embargo, las continuidades o semejanzas entre estas tres fuentes del yoga (FRAWLEY, 2012) pueden haberse debido a la contemporaneidad entre las mismas, y a que remiten a textos mas remotos como los Vedas, el Mahabharat (capítulo Mokshudharma) y los Upanishadas.

En el Gita el yoga se toma esencialmente como conjunción del alma (attā en Pali y ātman en Sanscrito) con una supuesta realidad absoluta y ultima, Dios y principio transcendental (Brahma). Es mas, Gita significa encomendarse a Dios. Sobresale la idea de un yoga devocional (adoración a Dios), en función del conocimiento directo de Brahma. Tal unión o fusión con Brahma se observa también en Patanjali, a pesar de que yoga significa también desunión con los obstáculos mentales. Si en el yoga del Gita se prescribe la repetición de la palabra "Gita", en los sutras de Patanjali se informan sobre la práctica de samadhi (cesación de la actividad mental), apelando a la repetición mantra OM, silaba expresión sonora de Dios (Ishwara).

Una de las sentencias de los sutras yogas de Patanjali es Yoga citta vritti nirodhah, que significa que el yoga se destina a la supresión de la mente o alma individual (citta). Citta, entendida como sustancia adopta diversas formas (vrittis), a saber, por ejemplo, las sensaciones o emociones placenteras y dolorosas. El yoga remueve esos vrittis, que obstaculizan la tranquilidad mental, causan dolor, ignorancia, egoísmo, apego, aversión y aferramiento a la vida (PATANJALI M. p.3). A tales efectos, en los sutras yogas de Patanjali se habla extensamente del control del ritmo de la respiración, lo que se denomina Prānāyāma (del Sanscrito). Se fija la mente en la inspiración y expiración, y, alternativamente se interrumpe, eliminándose las pausas (kumbhaka). La pacificación de chitta viene también de que el practicante medite en lo bueno y lo malo, la amistad, la compasión, la alegría, la felicidad. Varios pasajes de los sutras de Patanjali (véase lo referente al Kriya Yoga en el Libro II) recomiendan la mortificación del cuerpo con vistas a incrementar la fuerza de los órganos corporales y liberarlos de impurezas, y posibilitar que alcance cualidades como la mente, y responda a su control. Los sutras budistas no se comprometen con la perspectiva teísta del Gita y los sutras de Patanjali, y veremos que este diferendo doctrinal (filosófico, religioso o soteriológico) lleva a un diferendo técnico.

Usted no ve en los sutras MN. 10 o SN. 22 que se hable de mindfulness para tener experiencia de Dios. Kāyānupassanā desafía al Gita y los yogas sutras de Patanjali en este sentido. Tampoco que la revelación sea un argumento consistente para admitir la existencia de Dios. Incluso, en los sutras budistas tempranos se descarta que la idea de Dios haga falta para fundamental la moral (KARUNADASA, 2013, p. 154). No es difícil darse cuenta que la postulación de existencia de un Dios transcendental es inadmisible bajo las doctrinas budistas de paticcasamuppāda y anattā, donde, como se vio, se descarta que algo exista separado, permanente, y sin causas y condiciones.

Se reporta que Gautama en su empeño de búsqueda soteriológica aprende y domina a cabalidad técnicas de meditación de dos renombrados maestros yogas $\mathrm{Sa}$ mkhias. Primero, con Alara Kalama logra como se alcanza el estado, dimensión, esfera o absorción meditativa (jhana) denominada akincannayatana Ākiñcaññāyatana (meditado que se llega al estado de la nada). No suele tenerse exhaustiva descripción de este nivel meditativo, pero se enfatiza que Buda percibió que con tal práctica yoga no se logra el objetivo del nirvana. Se relata que el Buda pensó lo mismo respecto a la enseñanza yoga Uddaka Ramaputta sobre el jhana de nevasanna nasannayatan, es decir ni la percepción ni la no percepción. A pesar de su desafío al Brahmanismo ortodoxo, la corriente sramanista Sāmkhya (Samkhya) ${ }^{14}$, admite la idea de un alma eterna y el dualismo metafísico. Este diferendo doctrinal y a la vez técnico se deja ver muy bien en el sutra sobre la vacuidad (MN. 121) donde se habla de la vaciedad y la unidad dependiente de las esferas o jhanas de la no percepción y la nada. Y eso quiere decir, que con la doctrina y la técnica de los dos maestros Samkhyas el meditador sigue con apegos y no logra alcanzar el estado de mindfulness con respecto a esos estados de absorción meditativa.

Como vimos en el Gita y Patanjali se llama al control de la respiración. Pero en los sutras MN 10 y DN. 22 usted ve que un requerimiento técnico básico del mindfulness 
yoga es la no intervención en el fluir espontáneo de la respiración (WYNNE, 2007). Puede decirse que el control de la respiración expresa no solo una actitud que contrasta con el mindfulness, sino también con la perspectiva del no ser o no-ego en si mismo. Una vez mas se evidencia como las doctrinas que aquí se están analizando llevan a determinadas consecuencias técnicas y prácticas.

En los tiempos de Buda el mortificacionismo era muy usual, y ya vimos, incluso que los sutras yogas de Patanjali no lo descartan como recurso para la unión del alma con Brahma. Es más, en los yogas Sutras de Patanjali, se insiste en la posibilidad del yogui de separarse del cuerpo, de su invisibilidad cuando la receptividad ocular se interrumpe deliberadamente, y de la indestructibilidad de las cualidades corporales. Evidentemente, existe vínculo lógico e histórico entre la posición idealista y eternalista de la tradición Veda, Brahmanica e Hinduista, y la prácticas mortificativas adoptadas por algunas de sus vertientes, como la Samkhya. Sutras budistas descartan que aun en el estado de la nada la conciencia se separe del cuerpo (POSALA-MANAVA-PUCCHA, 2013). La persona que alcanza el estado de la nada, está plenamente consciente de cómo se origina y acontece ese estado. No obstante, la mortificacionismo no es una ideología unánime en la tradición védica. Y no se debe menospreciar que en la disciplina yogui busca potenciar el cuerpo con los elementos sutiles tales como los chakras o cakras (energías sutiles en el cuerpo) responsables tanto por la salud corporal como mental, y cualidades morales como el altruismo y la compasión.

Se ha insistido en que Kāyānupassanā cuando se dirige a la anatomía y fisiología del cuerpo tiene como intención el indicar que el cuerpo humano no es inherentemente atractivo. Se plantea también que en una ocasión cuando Buda habla ante un grupo de seguidores sobre los fluidos y el proceso de putrefacción del cuerpo, algunos que fervorosamente realizaron la práctica, terminaron cometiendo suicidio. (ANĀLAYO, 2003:149). Tal conexión es recurrentemente vapuleada en los sutras budistas, y se basa fundamentalmente en que al postularse que el ser es el alma y eterno, el cuerpo se verá como un obstáculo o algo que hay que hay que reducir a la mínima expresión. No cabria descartar que la apelación a la idea del cuerpo como algo repulsivo o repugnante haya sido un recurso pedagógico o misionero del Buda o del algún segmento de la comunidad inicial de monjes que el fundo. Empero, a decir verdad, en primer lugar, el postulado de annata desautoriza la asunción del cuerpo como algo que posee ser intrínseco. En segundo, lugar, incluso la doctrina Kāyānupassanā recalca que el meditador ha de mantenerse plenamente atento a la experiencia corporal en el momento presente, lo cual implica la suspensión de todo juzgamiento. En tercer lugar, de lo que se trata es de cultivar el desapego, y eso significa no apegarse a la experiencia que se presenta, ni en forma de deseo o placer ni en forma de aversión o repulsión.

Esto tiene relación con que Buda desaconsejó las prácticas yogas de austeridad y mortificación del cuerpo. De hecho se reporta que durante un tiempo antes de alcanzar el estado iluminado se entregó en cuerpo y alma a tales prácticas, exponiéndose, por ejemplo a sensaciones corporales y mentales dolorosas: parálisis, paro cardíaco, vómitos de sangre, etc., así como o el miedo, el desespero, la locura, los tormentos y lamentos. Se habla en los sutras de un ejercicio respiratorio donde el practicante siente estruendosos sonidos al entrar y salir el aire, lo cual le genera dolores no solo de oído, sino también de nariz, oído, cabeza y estómago. De hecho varios sutras se indica que la mortificación del cuerpo es un camino innoble y sin frutos para la meta de la salvación

Todo parece indicar que la comunidad budista temprana envistió la práctica mortificacionista de la comunidad Jain. En uno de los sutra budista (en el MN. 36) se registra que un discípulo de Mahavira, quien consolidó la secta o escuela Jaina, y sistematizó su filosofía, afirma que los discípulos de Buda se dedican a cultivar la mente (meditación), y no al cultivo del cuerpo (mortificación). Buda explica que el cuerpo y la mente están verdaderamente cultivados ni los dolores corporales ni mentales que pueden aparecer en la meditación se apoderan de la mente. No alcanzan al practicante ni el miedo ni el desespero ni los lamentos o tormentos, tampoco, la avidez, la codicia, o la avaricia. Cesa todo apego, aferramiento o atadura, pasión, ignorancia, y fabricaciones mentales. Cuando el cuerpo y la mente están cultivados el practicante no se identifica ni con su cuerpo ni con su mente ni con las sen- 
saciones corporales y mentales dolorosas o placentera ni con los conceptos y creencias.

Esta posición también va dirigida contras corrientes materialistas, que sugiere la identificación del alma humana con el cuerpo. Por lo tanto, cuanto este muere, ella se aniquila. Desde este punto de vista el esfuerzo moral no tiene sentido, y si la entrega a los placeres sensuales del cuerpo.

Las doctrinas budistas suelen presentarse como un "camino medio" entre el idealismo, dualismo, eternalismo, y mortificacionismo por una parte, y el materialismo, el aniquilacionismo, y el hedonismo por la otra. Uno puede observar como tal posición media se refleja en la práctica de Kāyānupassanā donde se orienta que el yogi concentre sin apego su atención al desvanecer del cuerpo (externa e interiormente), así como de las sensaciones, los objetos mentales, la consciencia, y cada uno de los cinco agregados del apego.

\section{Kāyānupassanā para atletas, pero....}

Ahora bien, amen de que el mindfulness budista responde a una expectativa soteriológica, hoy en día ha inspirado diversas perspectivas para el tratamiento de trastornos psicológicos y neurológicos. Resulta sobremanera recurrente la apelación a experimentos y test psicológicos o neurológicos aparatos de Prueba de Resonancia Magnética para demostrar que el mindfulness tienes efectos positivos: mejora la plasticidad neuronal, prevé y disminuye la depresión, aumenta el sentimiento de compasión y amabilidad y de felicidad en las personas. Investigaciones de la última década han demostrado que las actividades conscientes e inconcientes a las que se refiere la enseñanza budista dependen subalternamente de las actividades neuronales (en las amígdalas e hipocampos, y en las coaliciones de las sinapsis neuronales), es decir, que los procesos mentales y neuronales se correlacionan (co-arise) y se afectan mutuamente. De ahí que se proponga la práctica de "neurobhavana" (neuromeditación) o "neurodharma" con vistas a la erradicación del apego mediante la interiorización del sentimiento de satisfacción, la seguridad y la conexión (HANSON, 2013, p. 19).
En el campo de las llamadas Mindfullness-Based Therapies (KABAT-ZINN, 2013; SEGAL ET AL., 2012) se recomienda al meditador evocar mediante el lenguaje interior o monologo pensamientos positivos y no juzgatorios, y que se refieran al presente, no al pasado o futuro. Se llama a la evitar el uso de oraciones compulsivas ${ }^{15}$, que se estructuran con pronombres personales como "Yo" o "Mi". Más bien se prescribe meditar con pronombres impersonales de forma tal que disminuya el nivel de atribución personal y el sentido del ego ${ }^{16}$.

En el Budismo, como se vio, la frustración, desilusión, desesperanza son dukkha. Por ejemplo, dukkha es no poseer o perder lo que considera propio o valioso. En esta perspectiva cae un cuerpo que no se enferme nunca o lo menos posible, fuerte, rápido, o flexible, que nos permita alcanzar logros deportivos, reconocerse y ser reconocido como campeón o deportista de éxito. Cuando se frustra esta expectativa aparecen lamentos, tomentos, preocupaciones, penas, o el sentimiento de desdicha, desgracia, mala fortuna o mala suerte. Como se muestra en un trabajo recientemente, la visión budista del cuerpo y el trabajo de meditación que implica, ofrece subsidios a la Educación Física y los deportes (FREYRE, 2013). No es menos cierto que la identificación con el cuerpo, es recurrente en quienes practican deportes. $Y$ justamente los sutras MN 10 y SN 22 ofrecen la técnica yoga Kāyānupassanā con vistas a contrarrestar el apego al cuerpo y el apego al yo.

Los estudios sobre los efectos del mindfulness en el rendimiento físico y deportivo van in crescendo (MCCANN C., 2014). Digamos que Kāyānupassanā esta inspirando a sus aplicaciones actuales en este campo. De hecho ha surgido una disciplina denominada Mindful Sport Performance Enhancement (MSPE) que promueve en los atletas condiciones físicas y psíquicas favorables para el mejoramiento del desempeño deportivo. Se asegura que la práctica del mindfulness influye positivamente en condiciones como "attention" (atención), "affects" (afectos), "flow states" (estados de fluidez), y ciertos factores fisiológicos (TIMOTHY, ET AL., BERNIER AND FOURNIER, 2009).

Respecto a lo primero, con el desarrollo a través del mindfulness de una consciencia de no juzgar y centrada en 
el momento presente, se busca reducir la proclividad de distracción de la atención en los atletas antes las tareas que debe realizar. Se demanda que el mismo tenga la capacidad de prestar atención sostenida a las partes, componentes y sus de su cuerpo, así como al medio circundante al lugar de entrenamiento y de competencia. Tal atención puede se continua, situacional, de orientación, selectiva, flexible y analítica ("divided attention"). Se ha comprobado que las estrategias de atención asociativa garantizan un mejor desempeño deportivo que las contrarias (Ibidem, p. 17). El área de trabajo denominada "mindfulness-based conceptual model of attention" (modelo conceptual de la atención basado en el mindfulness) se centra precisamente en el cultivo de la atención en los atletas ${ }^{17}$. Con relación a lo segundo, el desarrollo de "enhance positive affect" busca reducir la "sport-related anxiety" (la ansiedad relacionada con el deporte), y desarrollar el "sport-related optimismo" (el optimismo relacionado con el deporte) (Ibidem, p.25), es decir, una valoración mas beneficiosa y positiva del momento presente ${ }^{18}$. En cuanto al tercer aspecto, los flow states se relacionan con lo que los psicólogos del deporte conceptualizan como "associate peak-performance experiences", o "being "in the zone". Es cuando el atleta disfruta de la situación, su cuerpo y su mente están en armonía, se presenta la aceptación y no reactividad respecto a los estados internos, están ausentes el pensamiento negativo y la duda. Por último, entre los factores fisiológicos se habla de la respuesta neurológica al dolor y la fatiga, la presión arterial sanguínea, los niveles de grasa, etc.

\section{Consideraciones finales}

El contenido de la exposición sobre Kāyānupassanā en los sutras MN. 10 y DN. 22 no se agota en la sección correspondiente por cuanto las demás prácticas (con las sensaciones, la mente y agregados) también se refieren al cuerpo. Por no hablar de que el mindfulness de la respiración implica a estos objetos.

Kāyānupassanā tributa a las doctrinas fundamentales del Budismo (Cuatro Nobles Verdades, el Camino Óctuple, los Cinco Agregados, el Surgir Dependiente, el No-Ser, y la Vacuidad). Indudablemente, se fundamenta en la vi- sión monista de la corporeidad donde estas doctrinas confluyen.

La encomienda soteriológica específica de Kāyānupassanā es la erradicación del apego al cuerpo y el apego al yo (o la identificación del yo con algo). Con esto se desafían a los yogas que se realizan bajo presupuestos idealistas, dualistas, metafísica, eternalistas y mortificacionista (como en el caso del yoga en los Samkhyas, el Gita, Patanjali) y jainas). El materialismo, el aniquilacionismo, y el hedonismo también se objetan porque promueven identificación del yo con el cuerpo.

Determinadas practicas espirituales llevan a una determinada teoría filosófica, y viceversa, es decir, las primaras cambian bajo el influjo de las segundas. Según un estudio reciente la corriente budista Yogācāra ofrece un ejemplo de lo primero y la enseñanza de la smrtyupasthāna en la Vajrayāna un ejemplo de lo segundo (WANGCHUK, 2013, p.24).

Lo mismo vale en el caso del contrapunteo entre el yoga budista y los otros yogas aquí mencionados. Ambas tradiciones yogas sugieren que el cuerpo no se reduzca a su dimensión física, que se atienda a las partes más sutiles del cuerpo, y que el cuerpo no se tome como mero objeto (MOFFIT, ET AL., 2008, p.2). Cuando sucede lo contrario, al yoga se le reduce a lo que se puede hacer con el cuerpo, $y$ al status de mero objeto.

Empero, no es menos cierto que las diferencias doctrinales llevan a diferencias técnicas. En el prānāyāma yoga de Patanjali se habla del control de la respiración, mientras en el ānāpānasati yoga budista se promueve lo contrario dado a que se sustenta en la doctrina de annata, es decir, del no-ser o no-yo.

Ciertamente, los sutras del Canon Pali del Budismo informan de técnicas yogas, pero no con tanta recurrencia y explicites como se puede verificar en el Gita y los yogas sutras de Patanjali. Quizás eso se explica porque el Budismo no se compromete no solo con la doctrina filosófica sino también con el proceder técnico de esos yogas.

Si se considera solo la visión del yoga que el Gita y los yogas sutras de Patanjali informan, entonces, no tiene sen- 
tido hablar de un yoga en el Budismo o un yoga budista. Mas bien cabria ver al Budismo como un desafiador del yoga. Pero si tenemos en cuenta que el yoga precede tanto a los unos como los otros, hablar de yoga budista perfectamente justificable.

Como se vio, el mindfulness budista sin duda alguna puede traer frutos a la Educación física y deportiva. Empero se corre el riesgo del no aprovechamiento del mensaje yoga budista en toda su potencialidad, cuando se sigue tomando el cuerpo como frontera de negociación de logros deportivos. No debe pasarse por alto que el Budismo propone un camino o practica moral. Obviamente, una persona deportivamente mindfull pudiera convertirse en una persona mindfull en la vida en general. El deporte pudiera ser un buen comienzo y un puente en esta dirección, siempre y cuando tribute a la formación de individuos con logros en uno y otro sentido. El caso "Oscar Pictorius", y de otros deportistas implicados en actos de violencia motiva pensar en este asunto.

El campo de "mindfulness y deportes" corre el riesgo de quedarse atrapado en la contribución del mindfulness al desempeño deportivo, y de no ir más allá, es decir, hacia donde nos invita el yoga del Budismo.

\section{Notas}

1 Mejora del Rendimiento Deportivo Mediante el Mindfulness.

2 Los sutras budistas tempranos Canon Pali se divide en tres partes. La de Sutras Nikayas se subdivide en Majjhima Nikaya (MN), Anguttara Nikāya (AN), Digha Nikaya (DN) y Samyutta Nikāya. Por eso a los sutras se les identifica por el corpus del Canon Pali al cual pertenecen y un número. Para este trabajo se ha utilizado principalmente la versión en español del Majjhima Nikaya (2006) traducida del pali por Amadeo Solé-Leris y Abraham Vélez de Cea. Los términos de pali y su correspondiente traducción al ingles los he tomado de la compilación (THE FOUR NOBLE TRUTH, 2008) hecha por Ānandajoti Bhikkhu.

3 En los sutras budistas tempranos se manejan sinónimos de Sati (mindfulness): annussati, que significa (calling to mind, "llamando a la mente"), patissati ( remembrance, recuerdo), dharanata (bearing in mind, colgado en la mente), saranata (recollection, recolección) (THERA, 2013, p.8).

4 Explicaciones e instrucciones sobre el "walking meditation" se ofrecen en Silananda (2010).

5 Visítese http://www.mindfulnessdc.org/bell/ para la instalación de este software.

6 Incluyendo las llamadas tales como las "fabricaciones" (papañca) corporales y mentales. Por tal se entiende cuando se con- sidera a los objetos sensoriales (visuales, olfatorios, sonoros, táctiles, y mentales) como placenteros o indeseables (BHIKKHU, 2010).

7 Quien redactó el sutra podía haber incluido este aspecto.

8 La traducción de dukkha como "sufrimiento" es predominante, aunque ampliamente discutida y desafiada por palabras como "humillación" "incertezas", "narcisismo innato" "insatisfacción" "perpetuar situación indeseable" o "empeorar más las cosas que lo que están". Un inventario extenso de definiciones de dukkha en ingles puede consultarse en "Dukkha" (2013).

9 Se tiene una traducción de este sutra del inglés al español en Libros Budistas.com (2000). Exposición extensa de esta doctrina se ofrece en varios sutras: en el MN 141 (Saccavibha冈ga sutta), MN. 26 (Aryaparisana sutta). El sutta DN. 22 presenta a Cattāri ariyasaccān como objeto del mindfulness.

10 El Budismo indica que el "mal moral" existe en tres niveles o estado: anuya (latencia), pariyutthana ("apareciendo"), vitikkama ("hiendo más allá"). Sila, samadhi y panna serían tres estadios del desarrollo moral llamados a contrarrestar esos tres niveles de proclividad o disposición psicológica al mal. El tercer nivel es el más peligroso (por su concreción, externalidad e impacto, pero más fácil de controlar. De ahí que postule que la práctica budista de la moral comienza con ese nivel (KARUNADASA, 2013, p. 13)

11 Explicación sustanciosa de los agregados puede encontrarse en Bhikkhu (2007).

12 En los sutras se plantea que la consciencia esta determinada por los demás agregados, pero, a su vez se plantea que la conciencia produce cuerpo ('mind-made body', mano-maya kaya), lo cual solo puede ser detectado por quien posea gran nivel meditativo (HARVEY, 1993, p.3).

13 La tesis del surgimiento dependiente desafía la idea de la causalidad linear e introduce la hipótesis de la retrocausalidad, es decir, que el afecto aparece primero que la causa. De eso se viene discutiendo en los estudios de Budismo y en Física quántica.

14 Se plantea que Patanjali fue influenciado por la doctrina yoga de esta corriente, y que en el Gita (Capitulo II) uno puede encontrar Samkhyas elementos (SHANKARDEV AND STEVENSON, 2007). No obstante, Samkhya no admite la existencia de un Dios creador (BERZIN, 2008), aunque si de un alma o espíritu universal. En sistema yoga Samkhyas consiste en ocho practicas (ashtanga yoga): yama (abstención), niyama (reglas de conducta), asnana (posturas), pranayana (control de la respiración), pratyahara (desconexion de los sentidos), dharana (concentración) y samathi (absorción del yo) (JARAYAM, 2014). En los yoga sutras de Patanjali se expone exactamente lo mismo.

15 Tales como "no puedo", "siento que el mundo esta en mi contra", "nadie me entiende", "no soy lo suficiente bueno", "nadie me entiende", "me siento no a gusto conmigo mismo", "algo no anda bien" (McCOWN et al., 2012, p. 10).

16 La verbalizar internamente los estados en términos impersonales implica por ejemplo decirse a si mismo: "este es el miedo", "esta es la tristeza", "este es el (LIEBERMAN, 2007; COLLINS ET AL., 2009, P. 3) Es mejor decirse "Se siente X" que decir "Me siento" o "Yo siento".

17 la atención a la audiencia constituye uno de los factores que mas distrae al atleta, y que expresa su proclividad a la identificación y apego al yo (CHINMOY SRI, 2010). 
18 La agresividad y la proclividad a hacer trampas es también una amenaza al deporte (WATANABE, 2000; GORNALL, 2004; LEWIS, 2008).

\section{Referencias}

BERZIN, A. (2008). Basic Tenets of the Samkhya and Yoga Schools of Indian Philosophy. June 2004, revised May 2008. http:// www.berzinarchives.com/web/en/archives/study/asian_nonbuddhist_traditions/indian_nonbuddhist_traditions/basic_tenets_samkhya_yoga_schools.html

FOUR NOBLE TRUTHS. (2008) Compiled by Ānandajoti Bhikkhu (mainly from Saccavibhamgasuttam, MN 141) http://www. ancient-buddhist-texts.net/Reference/Four-Noble-Truths.htm

BHIKKHU, B. The Buddha's Doctrine of Anatta: A Comparative Study of Self and Not-Self in Buddhism, Hinduism and Western Philosophy. Thailand: Buddhidamma Fund. Bangkok, 1990.

BHIKKHU, Th (2010). The Five Aggregates. A Study Guide. Access to insight http://www.accesstoinsight.org/lib/study/khandha.html

CHACHAKKA SUTTA (The Six Sextets) (1998) MN 148, translated from the Pali by Thanissaro Bhikkhu. Access to insight. http:// www.accesstoinsight.org/tipitaka/mn/mn.148.than.html

CHINMOY, S. Meditación y deportes. 2010. Disponible en: http:// www.srichinmoy.org/espanol/recursos/deporte/deporte_y_ meditacion/index.html. Acceso día 17 de agosto 2012.

COLLINS, S. E., Neharika, Ch., Sgaron, H., Grow, J, Otto, J, and Marlatt, A.G. (2009). Language based measures of Mindfulness: Initial validity and clinical utility. Psychol Addict Behav Retrieve from. http://www.ncbi.nlm.nih.gov/pmc/articles/ PMC2799300/

DHAMMACAKKAPPAVATTANA SUTTA: Setting the Wheel of Dhamma in Motion. (SN 56.11 PTS: S v 420 CDB ii) 1843, translated from the Pali by Thanissaro Bhikkhu. Access to Insight (2013) http://www.accesstoinsight.org/tipitaka/sn/sn56/ sn56.011.than.html

DUKKHA. (2013) edited by Access to Insight. Access to Insight (Legacy Edition), 5 November 2013. http://www.accesstoinsight. org/ptf/dhamma/sacca/sacca1/dukkha.html

ENCYCLOPEDIA OF HINDUISM. (2011). K.L. Sesghagiri, editor Chief. Indian Heretige Research Foundation in Association with Rupa and Co. Delhi.

FRAWLEY, D. (2012). Yoga and Buddhism: Similarities and Differences. The American Institute of Vedic Studies. http://vedanet. com/2012/06/13/yoga-and-buddhism-similarities-and-differences/

GORNALL, A. Is there a place for contact Sports in Buddhism. http://mingkok.buddhistdoor.com/en/news/d/21605 Acceso el día 27 de Julio de 2012.
HANSON, R. Neurodharma: Practicing with the Brain in Mind. International Conference Buddhist Meditative Praxis: Traditional Teachings \& Modern Application, 24 y el 25 de August, 2013. Hong Kong: Centre of Buddhist Studies. University of Hong Kong.

HARVEY, P. (1993). The mind-body relationship in Pali Buddhism: A philosophical investigation. Asian Philosophy, Vol. 3 No. 1 1993, pp.29-41 http://www.budsas.org/ebud/ebdha205.htm

JARAYAM, V. (2014). Samkhya and Yoga in Hinduism and Buddhism. Hinduwebsite. Com. http://www.hinduwebsite.com/ hinduism/philo/samkhyayoga.asp

KABAT-ZINN, J. (2013). Full Catastrophe Living: The Program of the Stress Reduction Clinic at the University of Massachusetts Medical Center. (2nd edition). New York: Dell Publishing.

KARUNADASA (2013). The Role of Meditation in the Threefold Scheme of Buddhist Mental Culture. International Conference Buddhist Meditative Praxis:Traditional Teachings \& Modern Application, 24 y el 25 de August, 2013. Hong Kong: Centre of Buddhist Studies. University of Hong Kong.

KAYAGATA-SATI SUTTA: Mindfulness Immersed in the Body. (2013). MN 119. S: M iii 88, translated from the Pali by Thanissaro Bhikkhuhttp://www.accesstoinsight.org/tipitaka/mn/ mn.119.than.html

LEWIS, W. Buddhism and Sports: via Sakyon Mipham Rinpoché. Disponible en http://www.elephantjournal.com/2008/11/buddhism- vs-sports-via-saky Acceso día 27 de marzo 2012.

LIBROS BUDISTAS.COM. Selección del Canon Pali. (2000) http:// www.librosbudistas.com/descargas/PAL.pdf

LIEBERMAN, M. D. (2007). Social Cognitive Neuroscience: A Review of Core Processes. California 90095-1563. Annu. Rev. Psychol. V. 58:259-89.

MAHA-NIDANA SUTTA (2013): The Great Causes Discourse (DN 15 PTS: D ii 55 ) translated from the Pali by Thanissaro Bhikkhu. Access to Insight (Legacy Edition), 30 November 2013,http:// www.accesstoinsight.org/tipitaka/dn/dn.15.0.than.html .

MCCANN C. (2014). The Application of Mindfulness Practice to Sport. 2014. http://www.thesportinmind.com/articles/theapplication-of-mindfulness-practice-to-sport/

McCOWN, D. and Reibel, D.M.M. (2012). Teaching Mindfulness. A Practical Guide for Clinicians and Educators. London, New York Dordrecht Heidelberg: Springer. Retrieve from http://www. mindfulnessstudies.com/wp-content/uploads/2014/01/1Teaching-Mindfulness.pdf

MILINDAPAÑHA SUTTA: The Questions of King Milinda (excerpts) (1890), translated from the Pali by T. W. Rhys Davids Part I of II. Volume XXXV of "The Sacred Books of the East" Oxford: The Clarendon Press. 
MOFFITT, Ph., Douglas A., Cope, and Powers S.(2008). Buddhism and Yoga: Where the Paths Cross. http://www.shambhalasun. com/index.php?option=com_content\&task=view\&id=1712

NATH, U. K. 2008. Early Buddhism and the Bhagavadgita. Delhi: Motilal Banasidass; New edition edition (February 5, 2008).

NHAT, H.Th. (1999). Vivir el Budismo. O la Práctica de la Atención Plena. Barcelona: Editorial Kairos.

PATANJALI, M. Los Yoga Sutras de Patanjali. Versión, traducción e interpretación de: Yogacharya Dr. Fernando Estévez Griego (Dharmachari Swami Maitreyananda). http://www.yogaintegral.biz/YogaSutrasdePatanjali.pdf

PINEAU, T. R.P., Carol R. G, \& Keith A. K. Mindfulness in Sport Performance. In A. le, C. Ngnoumen, \& E. Langer (Eds.), Handbook of mindfulness. Oxford, UK: Wiley-Blackwell. http://psychology.cua.edu/res/docs/Pineau-Glass-Kaufman-Mindfulness-inSport-Performance-revised.pdf

POSALA-MANAVA-PUCCHA (2013): Posala's Questions (Sn 5.14 PTS: Sn 1112-1115) translated from the Pali by Thanissaro Bhikkhu. Access to Insight (Legacy Edition), 30 November 2013,http://www.accesstoinsight.org/tipitaka/kn/snp/ snp.5.14.than.html

POTTHAPADA SUTTA: About Potthapada (DN 9), translated from the Pali by Thanissaro Bhikkhu. (2013). Access to Insight (Legacy Edition), 30 November. Retrieve from http://www.accesstoinsight.org/tipitaka/dn/dn.09.0.than.html

SEGAL, Z.V., Williams, J. M.G., \& Teasdale, J. D. (2012). Mindfulness-Based Cognitive Therapy for Depression (2nd edition). New York: Guilford.

SILANANDA, S.U. (2010). "The Benefits of Walking Meditation". Access to Insight (Legacy Edition), 7 June 2010.http://www.accesstoinsight.org/lib/authors/silananda/bl137.htm

THE SATIPATTHANA SUTTA translated from the Pali by Anālayo, in Anālayo's Satipatthāna: The Direct Path to Realization. Birmingham: Windhorse Pub, 2003.

THERA, S. (2013). The Way of Mindfulness. The Satipatthana Sutta and Its Commentary http://www.accesstoinsight.org/lib/ authors/soma/wayof.html

THICH Nhat, H. Th. ( A Guide to Walking Meditation http://www. dhammatalks.net/Books2/Thich_Nhat_Hanh_A_Guide_to_ Walking_Meditation.htm

WANGCHUK, D. (2013). Apropos the Problems of the Theory-Praxis Dichotomy in Buddhism: The Case of the Four Applications of Mindfulness (Smrtyupasthāna: satipattthāna) in Vajrayāna. International Conference Buddhist Meditative Praxis:Traditional Teachings \& Modern Application, 24 y el 25 de August, 2013. Hong Kong: Centre of Buddhist Studies. University of Hong Kong.
WATANABE, T. Buddhism and Basketball? It Works for Phil Jackson. Los Angeles Times. June/2000. Disponible en http://articles

WYNNE, A. The Origin of Buddhist Meditation London \& New York: Routledge, 2007.

Data de recebimento 05/07/2014

Data de aprovação 10/08/2014

Data de aprovação 29/08/2014 